\title{
An unusual pre-ligamentous thenar motor branch of the median nerve
}

\author{
Mohammad M. Al-Qattan ${ }^{1,2} \cdot$ Khalid Al-Zahrani ${ }^{3}$
}

Received: 12 December 2016 / Accepted: 26 December 2016/Published online: 13 January 2017

(C) Springer-Verlag Berlin Heidelberg 2017

\begin{abstract}
The pre-ligamentous variant of the thenar motor branch (TMB) of the median nerve is extremely rare. In all previously reported cases, the branch arose from the radial or antero-radial aspect of the median nerve in the distal forearm and then pierced the antebrachial fascia to reach the thenar muscles. We report on a case in which the pre-ligamentous TMB not only arose from the ulnar side of the median nerve but it also remained deep to both the antebrachial fascia and the transverse carpal ligament until it reached the thenar muscles. The course of this variant puts the TMB at significant risk of injury during both open and endoscopic carpal tunnel release. Level of Evidence: Level V, risk study.
\end{abstract}

Keywords Thenar motor branch $\cdot$ Median nerve $\cdot$ Persistent median artery

Mohammad M. Al-Qattan

moqattan@hotmail.com

1 Department of Surgery, King Saud University, 18097, Riyadh 11415, Saudi Arabia

2 National Hospital (Care), Riyadh, Saudi Arabia

3 Department of Surgery, King Saud University, Riyadh, Saudi Arabia

\section{Introduction}

Knowledge on the anatomical variations of the thenar motor branch (TMB) of the median nerve is essential, in order to avoid its iatrogenic injury during carpal tunnel release [1-3]. Classification of these variations is generally based on the site of origin of the TMB from the median nerve. In the extra-ligamentous type (seen in about $75 \%$ of the population) [3], the TMB arises distal to the transverse carpal ligament and then takes a retrograde course to the thenar muscles. In both the sub- and trans-ligamentous types (seen in about 13 and $11 \%$ of the population, respectively), the TMB arises within the carpal tunnel (under the transverse carpal ligament). In the former sub-ligamentous type, the TMB remains deep to the transverse carpal ligament until it reaches the thenar muscles. In the latter trans-ligamentous type, the TMB pierces the ligament to reach the thenar muscles. Finally, the pre-ligamentous TMB (very rare) [3] arises from the median nerve proximal to the transverse carpal ligament and then pierces the antebrachial fascia of the distal forearm to reach the thenar muscles [4].

Anatomical variations of the TMB may also be classified according to the side of branching from the median nerve. In about $98 \%$ of the population, the TMB arises from the radial or antero-radial aspect of the median nerve [3]. In the remaining $2 \%$ of the population, the TMB arises from the ulnar side of the median nerve [3].

In this paper, the authors document a previously undescribed variant of pre-ligamentous TMB in which it arose from the ulnar side of the median nerve in the distal forearm 
and remained deep to the transverse carpal ligament without piercing the antebrachial fascia to reach the thenar muscles. This variant is not only extremely rare but also puts the nerve at significant risk of iatrogenic injury during carpal tunnel release.

\section{Case report}

A 30-year-old man sustained a glass injury to the distal forearm resulting in a $3-\mathrm{cm}$ longitudinal laceration in the mid-forearm along the course of the median nerve. Severe arterial bleeding was noted, which was controlled by a tight bandage at the workplace. At the time of presentation to our hospital, there was still active bleeding, despite the tight bandage. The patient was immediately taken to the operating room.

Following endotracheal intubation, an arm tourniquet was applied and inflated before removing the tight bandage. Wound exploration along with carpal tunnel release was performed. The source of bleeding was from a partially lacerated persistent median artery, which ran
Fig. 1 a An intraoperative view. b The diagrammatic representation of the findings in our case (see text for details)
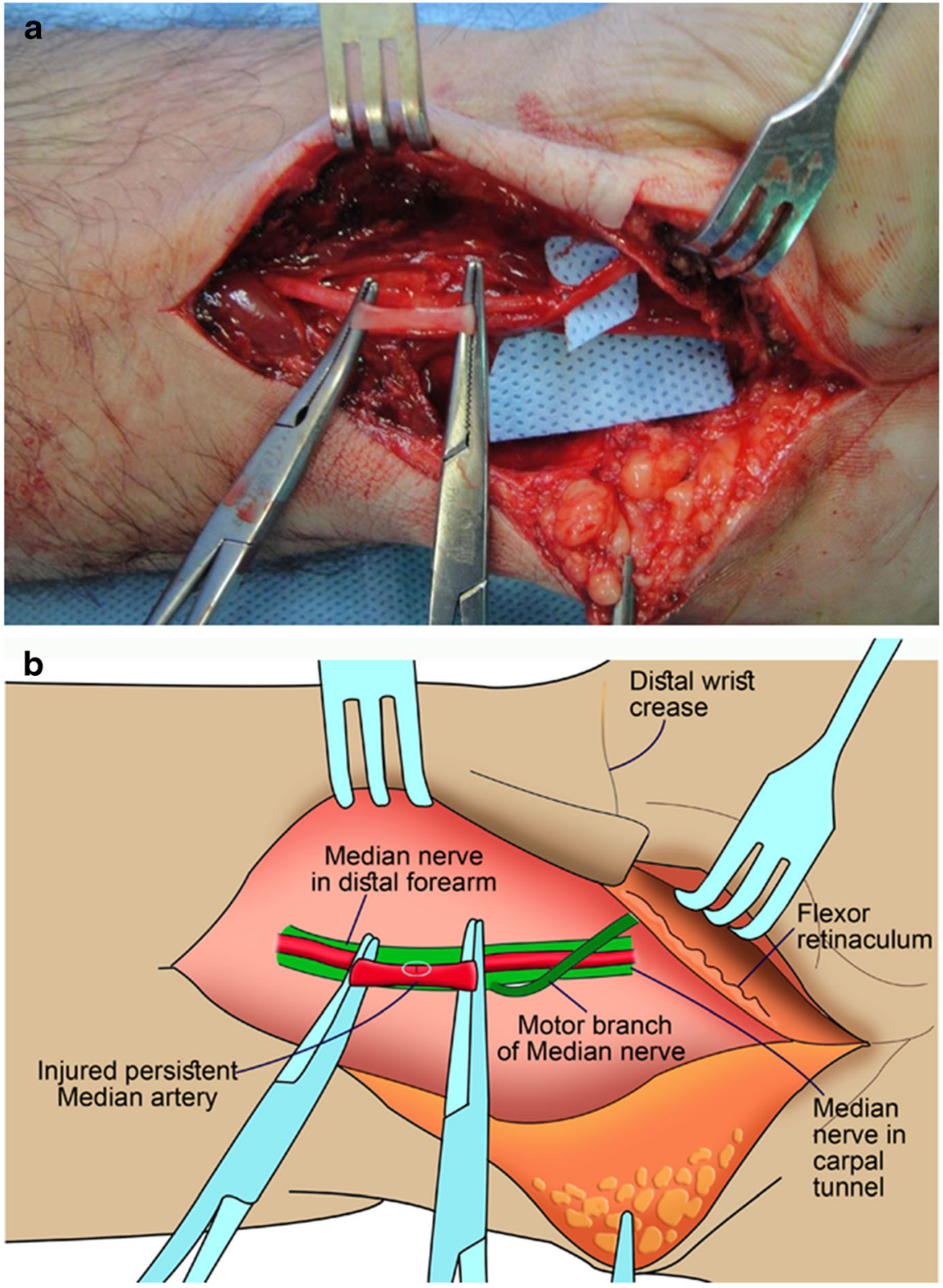
on the anterior aspect of the median nerve. There was no injury to the median nerve or flexor tendons. A very unusual variant of the TMB was noted intraoperatively (Fig. 1a, b). The TMB arose from the ulnar side of the median nerve $2 \mathrm{~cm}$ proximal to the distal wrist crease. The TMB remained deep to both the antebrachial fascia and the transverse carpal ligament, crossing (from ulnar to radial) the persistent median artery to reach the thenar muscles. Nerve stimulation resulted in thenar muscle contraction. The injured persistent median artery was transected and ligated after the release of the tourniquet to ensure an adequate vascularity of all digits. Both radial and ulnar arteries had strong palpable pulses at the wrist. Prior to ligation of the persistent median artery, a vascular clamp was applied to the injured median artery and a sterile pulse oximetry was applied to the pulps of the digits. Oxygen saturation was $99-100 \%$ in all digits with a normal pulse wave, and hence, repair of the median artery was not necessary. The postoperative course was uneventful with return to work 3 weeks after injury.

\section{Discussion}

The current case has a combination of rare variations: a persistent median artery (seen in about $10 \%$ of the population) [5], an ulnar origin of the TMB (seen in $2 \%$ of the population) [3], and a pre-ligamentous TMB variant (very rare) [3]. In all previously reported cases of the pre-ligamentous variants, the TMB arose from the radial or antero-radial aspect of the median nerve in the distal forearm and then pierced the antebrachial fascia to run superficial to the transverse carpal ligament until it reached the thenar muscles $[3,4,6]$. Our case was unique because the pre-ligamentous TMB not only arose from the ulnar side of the median nerve but it also remained deep to both the antebrachial fascia and the transverse carpal ligament until it reached the thenar muscles. The course of this variant puts the TMB at significant risk of injury during both open and endoscopic carpal tunnel release.

The presence of a persistent median artery in our case is also interesting. Several authors noted that a persistent median artery is frequently associated with a high division of the median nerve (also known as the bifid median nerve) $[1,3,7]$. A bifid median nerve is only seen in $2.6 \%$ of the population; but it is associated with a $63 \%$ prevalence of a persistent median artery [3].
Lanz [1] noted that the two parts of bifid median nerves are usually equal in size. Other authors documented a larger radial division of the bifid median nerve [8]. Hence, the presence of a persistent median artery in our case may actually represent a concurrent high division of the median nerve: a small ulnar division representing a pre-ligamentous TMB and a large radial division containing the remaining trunk of the median nerve.

Finally, it is important to be aware that the persistent median artery may be the main blood supply to the radial two digits [7-9]. Therefore, hand vascularity should be checked before transection or excision of the persistent median artery [7], and this was done in our case.

Acknowledgements We thank our artist for drawing Fig. 1b.

\section{Compliance with ethical standards}

Conflict of interest Mohammad M. Al-Qattan and Khalid Al-Zahrani declare that they have no conflict of interest.

Patient consent The patient gave written consent before his inclusion in this study.

Funding The study is funded by the College of Medicine Research Center, Deanship of Scientific Research, King Saud University, Riyadh, Saudi Arabia.

Ethical approval For this type of study formal consent is not required.

\section{References}

1. Lanz U (1997) Anatomical variations of the median nerve in the carpal tunnel. J Hand Surg Am. 2:44-53

2. Kozin SH (1998) The anatomy of the recurrent branch of the median nerve. J Hand Surg Am. 23:852-858

3. Henry BM, Zwinczewska H, Roy J et al (2015) The prevalence of anatomical variations of the median nerve in the carpal tunnel: a systematic review and meta-analysis. PLoS One 10(8): e0136477

4. Al-Qattan MM (2010) Variations in the course of the thenar motor branch of the median nerve and their relationship to the hypertrophic muscle overlying the transverse carpal ligament. J Hand Surg Am 35: $1820-1824$

5. Coleman SS, Anson BJ (1961) Arterial patterns in the hand based upon a study of 650 specimens. Surg Gynecol Obstet 113:409-424 
6. Raviprasanna KH, Poornima GC, Dakshayani KR (2014) Study of branching pattern of median nerve at carpal tunnel and its importance in surgical anatomy. IJBR 05(09). doi:10.7439/ijbr

7. Al-Qattan MM, Al-Zahrani K, Al-Amawi M (2009) The bifid median nerve re-visited. J Hand Surg Eur Vol 34:212-214

8. Schultz RJ, Endler PM, Huddlerston HD (1973) Anomalous median nerve and an anomalous muscle belly of the first lumbrical associated with carpal tunnel syndrome. J Bone Joint Surg Am 55:1744-1746

9. Beran SJ, Friedman RM, Kassir M (1997) Recurrent digital ischaemia due to thrombosis of the persistent median artery. Plast Reconstr Surg 99:1169-1171 